\title{
Validação de escalas diagnósticas do funcionamento familiar para utilização em serviços de atenção primária à saúde ${ }^{1}$
}

\author{
Olga G. Falceto, ${ }^{2}$ Ellis D. Busnello ${ }^{3}$ e Mary C. Bozzetti ${ }^{4}$
}

\begin{abstract}
RESUMO O atendimento a pacientes em serviços primários de saúde deveria compreender uma avaliação tanto física como psicológica; em muitos casos, o sucesso do tratamento depende da compreensão do indivíduo e de suas interações, em especial de sua interação na família. Portanto, é importante instrumentalizar os profissionais de saúde para que sejam capazes de trabalhar com os indivíduos nesse contexto social. Estudamos três escalas diagnósticas do funcionamento familiar consagradas na literatura internacional (FACES III, Beavers-Timberlawn e GARF), procurando validá-las para uso em nosso meio (Porto Alegre, Brasil). Comparamos os resultados do preenchimento das escalas com a avaliação clínica, feita através de entrevista familiar semi-estruturada, em 31 famílias clínicas. A escala auto-respondida FACES III também foi testada em 102 famílias na comunidade. A escala FACES III mostrou uma correlação linear positiva, porém fraca, entre coesão familiar e risco para doença mental, mas não em relação à adaptabilidade. As escalas BT e GARF demonstraram forte correlação positiva com a avaliação clinica. A escala FACES III não demonstrou ser adequada para uso em triagem de cuidados primários; entretanto, as escalas BT e GARF mostraram-se muito úteis na formulação e classificação do diagnóstico familiar.
\end{abstract}

$\mathrm{O}$ atendimento a pacientes que procuram serviços primários de saúde deveria compreender uma avaliação global, tanto física como psicológica. Diversos estudos internacionais sobre prevalência de transtornos mentais entre pacientes atendidos em serviços de saúde que prestam cuidados primários (1) mostram que de 10 a 31\% dos

\footnotetext{
1 Trabalho baseado na tese de mestrado intitulada "Famílias com desenvolvimento funcional e disfuncional: validação das escalas diagnósticas Faces III, Beavers-Timberlawn e Avaliação Global do Funcionamento Interacional (GARF)", apresentada em 1997 ao curso de pós-graduação em Medicina: Clínica Médica da Universidade Federal do Rio Grande do Sul (UFRGS).

2 Universidade Federal do Rio Grande do Sul, Faculdade de Medicina, Departamento de Psiquiatria,
}

pacientes apresentam pelo menos um tipo de diagnóstico psiquiátrico (principalmente depressão e ansiedade). Além disso, sabe-se que as dificuldades nas interações sociais podem manifestar-se sob a forma de doenças psicossomáticas, problemas emocionais ou de relacionamento, de forma isolada ou em combinação $(2,3)$.

Porto Alegre, RS, Brasil. Correspondência e pedidos de separatas devem ser enviados a esta autora no seguinte endereço: Rua João Abbott 451, conjunto 402, CEP 90460-150, Porto Alegre, RS, Brasil. Telefone/fax: +55-51-332-1211. E-mail: ovidolg@ uol.com.br

3 Universidade Federal do Rio Grande do Sul, Curso de Pós-Graduação em Clínica Médica.

4 Universidade Federal do Rio Grande do Sul, Departamento de Medicina Social.
O desenvolvimento de problemas psiquiátricos é influenciado pelas particularidades do indivíduo e de suas interações com a família, sendo que as dificuldades são maiores quando já há outros membros da família com doença psiquiátrica. $\mathrm{O}$ processo de adoecimento também é afetado pelas relações de vizinhança, de trabalho e pelas relações do indivíduo com instituições como a escola e os centros de saúde.

Para muitos pacientes, o sucesso do tratamento depende do entendimento da dinâmica familiar e da função que a doença assume na família e na vida do próprio paciente. $\mathrm{O}$ envolvimento da família no tratamento de todas as manifestações de uma doença, sejam elas de caráter psiquiátrico ou não, fre- 
qüentemente torna a intervenção mais efetiva e multiplica os recursos de auxílio ao paciente, em especial sua adesão ao tratamento (4-6).

Por ser o funcionamento familiar tão influente na proteção da saúde ou na manifestação de doenças, torna-se importante instrumentalizar os profissionais que estão na linha de frente, prestando atendimento à maioria das pessoas que procuram o sistema de saúde, para que possam reconhecer os sinais de disfunção e as áreas de capacitação da família, e dar-lhes recursos básicos para poder identificar famílias que necessitam atenção especial.

Embora o estudo da dinâmica familiar seja uma área de trabalho multidisciplinar, a sistematização do conhecimento tem sido realizada por psiquiatras e psicólogos que vêm trabalhando na montagem de um sistema de diagnóstico do funcionamento familiar. O Manual de Diagnóstico e Estatística dos Distúrbios Mentais (DSM-IV) (7) e a décima revisão da Classificação Internacional de Doenças e Causas de Morte (CID-10) (8), largamente utilizados em psiquiatria, incluem a avaliação de fatores psicossociais como estressores, mas ainda não privilegiam a avaliação da estrutura e funcionamento da família para fins diagnósticos. Entretanto, a avaliação diagnóstica da família tem sido feita mediante a utilização de questionários $(9,10)$. Entre esses, a Escala de Avaliação da Adaptabilidade e Coesão Familiar, conhecida como FACES III (Family Adaptability and Cohesion Evaluation Scales) $(11,12)$ é o questionário mais estudado, por ser autoaplicado, breve e simples, com uma estrutura que seria adequada para uso como instrumento de triagem em serviços de atenção primária à saúde. A escala de Beavers-Timberlawn (BT) (4) e a Avaliação Global do Funcionamento Relacional (Global Assessment of Relational Functioning, GARF) (10) estão também entre as mais utilizadas nas pesquisas internacionais.

$\mathrm{O}$ presente estudo foi dividido em duas etapas. Inicialmente, aplicou-se a escala FACES III em uma amostra populacional na tentativa de validá-la para o contexto local, reproduzindo a metodologia dos estudos publicados na literatura internacional. Percebemos, no entanto, a necessidade de comparar a escala com um padrão-ouro para efetivar a validação. Assim, este trabalho descreve, também, a validação das escalas FACES III, BT e GARF em uma amostra heterogênea, utilizando como padrão-ouro a avaliação clínica.

\section{MATERIAIS E MÉTODOS}

Foram realizados dois estudos transversais (A e B) planejados para validar três instrumentos diagnósticos do funcionamento familiar (FACES III, BT e GARF) (anexo 1). A entrevista clínica foi utilizada no estudo A como padrão-ouro para validação dos instrumentos.

O estudo A incluiu 31 famílias e o estudo B, 102 famílias. Ambas as amostras eram de famílias constituídas por um casal em seu primeiro casamento ou em união posterior, nesse caso com duração de pelo menos 5 anos, e seus filhos (famílias nucleares completas). Nenhum membro da família apresentava doença orgânica, nem problemas com a lei nos últimos 3 anos. Na amostra A foram incluídas, consecutivamente, famílias que procuraram o serviço psiquiátrico, além de seis famílias voluntárias que se ofereceram para o estudo por indicação de colegas. A amostra A incluiu, portanto, portanto, algumas famílias portadoras de problemas psiquiátricos. A amostra B arrolou, por amostragem domiciliar aleatória sistemática, famílias de uma comunidade socioeconomicamente heterogênea, localizada próximo a um hospital universitário.

No estudo A foram aplicados os três instrumentos diagnósticos e a entrevista clínica. No estudo B, que foi o estudo exploratório inicial, aplicou-se apenas a escala FACES III.

A escala FACES III (auto-respondida) é composta de 20 perguntas. As 10 perguntas de número ímpar dizem respeito à coesão familiar, à capacidade da família de manter-se unida frente às vicissitudes do dia-a-dia. As 10 perguntas pares referem-se à adaptabilidade familiar, ou seja, à capaci- dade dos membros da família de modificar papéis e regras de funcionamento para adequá-los à tarefa ou ao momento a enfrentar. As perguntas são expressas de forma simples para poderem ser entendidas e respondidas, inclusive por analfabetos (neste caso, o entrevistador lê em voz alta as perguntas, sem interpretá-las) e adolescentes maiores de 12 anos. Por esta razão, em princípio, esta escala poderia ser utilizada para triagem em cuidados primários. O objetivo é obter respostas de todos os membros da família, de forma que estejam representadas, no resultado, as várias percepções do funcionamento da família. Olson descreve um modelo de análise dos resultados, o "modelo circumplexo", que resulta de diferentes combinações das dimensões coesão e adaptabilidade $(11,12)$. Sumariamente, o modelo circumplexo permite classificar as famílias em 16 tipos, combinando os parâmetros coesão e adaptabilidade. Estes 16 tipos, por sua vez, são classificados em três grupos de risco para desenvolvimento de doenças psiquiátricas: baixo, moderado e alto. Do grupo de baixo risco fazem parte as famílias com coesão e adaptabilidade médias; do grupo de risco moderado, famílias com um dos parâmetros médio e o outro extremo; e do grupo de alto risco, famílias em que tanto a coesão quanto a adaptabilidade situam-se nos extremos.

A escala BT (completada pelo entrevistador a partir de observação) especifica várias características da estrutura familiar, do exercício da autonomia e da expressão do afeto na família. Descreve várias possibilidades diagnósticas e solicita do avaliador notas para cada tópico, além de uma nota global do funcionamento da família, de 1 a 10 , onde a nota de maior risco para desenvolvimento de doenças psiquiátricas é 10 .

A escala GARF (completada pelo entrevistador a partir de observação) já está incluída como apêndice no DSM-IV. Seu uso tende, portanto, a tornar-se generalizado. A escala GARF descreve de forma simples diversas situações e permite ao avaliador dar uma nota global ao funcionamento familiar, de 1 a 99. Quanto melhor o funcionamento da família, maior é a nota. 
Com base na descrição oferecida pelos instrumentos (anexo 1), definimos os seguintes pontos de corte para classificação de risco de funcionamento familiar: para a escala BT, o grupo de alto risco tinha pontuação $\geq 9$; o grupo de risco moderado, $\geq 5$ e $\leq 8$; e o grupo de baixo risco, $\leq 4$. Para a escala GARF considerou-se grupo de alto risco aquele com pontuação $\leq 50$; de risco moderado, $\leq 80$ e $\geq 51$; e de baixo risco, $\geq 81$. Na escala FACES III, os grupos de risco foram classificados de duas formas: segundo o modelo circumplexo e segundo o modelo linear, ou seja, analisando separadamente as características coesão e adaptabilidade familiar.

A tradução dos instrumentos, após a autorização dos seus autores, foi realizada independentemente por três pessoas proficientes em inglês. Depois disso, as escalas em português foram traduzidas novamente para inglês por outras pessoas, para garantir sua precisão. A seguir, os instrumentos foram testados em um estudo piloto, com o objetivo de adequar a linguagem à nossa população.

Utilizamos uma medida qualitativa, a entrevista clínica semi-estruturada, como padrão-ouro por ser, no momento, o melhor método de avaliação da complexa interação familiar, na tentativa de encontrar, através do estudo comparativo, um instrumento quantitativo simples capaz de avaliar especificamente o funcionamento familiar.

As entrevistas foram realizadas por psiquiatra treinado em terapia familiar e tiveram duração entre 60 e 90 minutos. Delas participaram sempre todos os membros da família nuclear. Na primeira etapa da entrevista, as comunicações espontâneas permitiram observar quem falava e quais temas e interações surgiam, facilitando a entrada na segunda etapa da entrevista, que examinou sistematicamente os papéis desempenhados pelos membros, as relações entre eles, e os hábitos e rotinas familiares. Ao mesmo tempo, examinou-se como e quem dava as informações, quais as diferenças de opinião entre os membros da família, qual era o clima emocional da sessão, se existiam divergências entre o casal (explícitas ou implícitas), como as crian-

TABELA 1. Características demográficas das amostras estudadas em termos do risco para desenvolvimento de doenças psiquiátricas, Porto Alegre (RS), Brasil, 1997

\begin{tabular}{|c|c|c|c|c|c|}
\hline \multirow[b]{2}{*}{ Variável } & \multicolumn{3}{|c|}{ Estudo $A^{a}$} & \multirow[b]{2}{*}{$\begin{array}{l}\text { Estudo B } \\
(n=102)\end{array}$} & \multirow[b]{2}{*}{$P$} \\
\hline & $\begin{array}{c}\text { Baixo risco } \\
\quad(n=9)\end{array}$ & $\begin{array}{c}\text { Risco } \\
\text { moderado } \\
(n=13)\end{array}$ & $\begin{array}{l}\text { Alto risco } \\
(n=9)\end{array}$ & & \\
\hline Número de filhos ${ }^{b}$ & $2,00 \pm 1,12$ & $1,77 \pm 1,09$ & $2,89 \pm 1,05$ & $2,34 \pm 1,83$ & 0,22 \\
\hline Idade do paib & $39,00 \pm 8,15$ & $40,77 \pm 9,63$ & $50,22 \pm 7,89$ & $47,58 \pm 14,79$ & 0,104 \\
\hline Idade da mãeb & $35,78 \pm 8,80$ & $38,62 \pm 10,02$ & $48,44 \pm 7,57$ & $43,24 \pm 13,51$ & 0,114 \\
\hline Idade dos filhos ${ }^{b}$ & $10,74 \pm 6,14$ & $9,00 \pm 5,78$ & $15,58 \pm 7,34$ & $19,60 \pm 12,18$ & 0,000 \\
\hline Renda familiarc & $25,81 \pm 24,64$ & $35,24 \pm 48,91$ & $27,23 \pm 18,09$ & $34,22 \pm 36,86$ & 0,87 \\
\hline Variação da renda familiard & $0-55,00$ & $0-180,00$ & $0-60,00$ & $0-213,90$ & \\
\hline
\end{tabular}

a Categorização de risco de acordo com a avaliação clínica.

${ }^{\mathrm{b}}$ Média \pm desvio padrão.

c Média da renda familiar em salários mínimos \pm desvio padrão.

d Amplitude da variação da renda em salários mínimos (um salário mínimo $\cong$ US $\$ 70,00$ ).

ças participavam, se pareciam envolvidas nos problemas do casal e se seu desenvolvimento psicológico era adequado à sua idade cronológica. Investigou-se também a história do casal e da família e a história intergeracional, procurando conhecer o relacionamento e possíveis padrões de repetição. Por último, foram abordados pontos potencialmente mais conflituosos, tais como manejo de dinheiro, para avaliar a capacidade da família de prevenir e resolver problemas.

Após a entrevista, todos os membros da família com mais de 12 anos preenchiam o questionário FACES III. $\mathrm{O}$ entrevistador preenchia as escalas GARF e BT. As famílias foram avaliadas e classificadas, segundo a impressão clínica, em três níveis de funcionamento familiar: baixo risco, risco moderado e alto risco para o desenvolvimento de transtorno psiquiátrico.

A análise estatística das informações coletadas incluiu a comparação dos instrumentos com a avaliação clínica. Análise de variância e teste de Duncan foram utilizados para comparar as categorias de risco. As variáveis numéricas sem distribuição normal foram analisadas através dos testes de Kruskal-Wallis e Mann-Whitney. Para verificar a correlação entre variáveis numéricas ou ordinais, utilizou-se o coeficiente de correlação de Pearson e Spearman. A concordância entre variáveis categóricas foi testada através do coeficiente de concordância Kappa.
O nível de significância estabelecido foi $P<0,05$.

\section{RESULTADOS}

A tabela 1 apresenta algumas variáveis demográficas das amostras estudadas, que incluíram famílias em todas as fases do ciclo vital, desde casais ainda sem filhos até famílias com filhos adultos que haviam saído de casa, e de todas as classes sociais. A comparação entre as duas amostras foi significativa apenas para idade dos filhos, que foi maior nas famílias do estudo B.

Os resultados da aplicação das escalas FACES III foram inicialmente analisados segundo o modelo circumplexo descrito por Olson $(11,12)$ (tabela 2). Nossos resultados foram comparados com os do estudo inicial de Olson, nos Estados Unidos, e com os de um estudo colombiano. ${ }^{5}$ Os achados de nosso estudo mostraram que $61 \%$ da população, que se autodefiniram como sem problemas psiquiátri$\cos$, apresentavam risco para doença mental de moderado a alto. Ao compararmos nossos dados com os dos outros estudos, não observamos diferenças estatisticamente significativas quanto à proporção de indivíduos

\footnotetext{
Córdoba AH. Familias en clínicas de Bogotá — su funcionamiento según el modelo circumplejo de D.H. Olson [monografia]. Bogotá: 1989.
} 
TABELA 2. Freqüência populacional por grupo de risco para desenvolvimento de doenças psiquiátricas no presente estudo e em dois estudos internacionaisa

\begin{tabular}{lcccc}
\hline \multicolumn{1}{c}{ Risco } & $\begin{array}{c}\text { Estudo B } \\
n=102 \\
(\%)\end{array}$ & $\begin{array}{c}\text { Olson }^{\mathrm{b}} \\
=1140 \\
(\%)\end{array}$ & $\begin{array}{c}\text { Córdoba }^{\mathrm{c}} \\
n=203 \\
(\%)\end{array}$ & $P$ \\
\hline Baixo risco & 39 & 49 & 53 & \\
Risco moderado & 48 & 40 & 39 & 0,149 \\
Alto risco & 13 & 11 & 7 & \\
\hline
\end{tabular}

${ }^{a}$ Classificação em grupos de risco utilizando o modelo circumplexo.

b Olson et al. (12).

' Córdoba AH. Familias no clínicas de Bogotá - su funcionamiento según el modelo circumplejo de D.H. Olson [monografia]. Bogotá: 1989.

TABELA 3. Risco para desenvolvimento de doenças psiquiátricas conforme o questionário FACES III e a entrevista familiar no estudo A, Porto Alegre (RS), Brasil, 1997

\begin{tabular}{|c|c|c|c|}
\hline Risco & $\begin{array}{l}\text { Classificação pelo } \\
\text { FACES III } \\
(n=31) \\
(\%)\end{array}$ & $\begin{array}{l}\text { Classificação pela } \\
\text { avaliação clínica } \\
\left(\begin{array}{c}n=31) \\
(\%)\end{array}\right.\end{array}$ & $P^{\mathrm{b}}$ \\
\hline Baixo risco & 43 & 29 & \\
\hline Risco moderado & 50 & 42 & 0,27 \\
\hline Alto risco & 7 & 29 & \\
\hline
\end{tabular}

a Conforme o modelo circumplexo de Olson.

${ }^{\mathrm{b}}$ Kappa.

classificados nas diversas categorias de risco.

A tabela 3 descreve os resultados da comparação entre os grupos de risco classificados pela escala FACES III em relação à amostra $\mathrm{A}$, segundo o modelo circumplexo e pela avaliação através da entrevista clínica. Houve concordância baixa e não estatisticamente significativa entre as duas avaliações (kappa $=0,07 ; P=0,27$ ).

Seguindo a experiência internacional, testamos, no estudo A, outro tipo de análise da escala FACES III. Clinicamente, está cada vez mais claro que existe uma correlação linear positiva entre coesão e bom funcionamento familiar e isso também parece válido para a adaptabilidade familiar. Nossos resultados (tabela 4) demonstraram correlação linear positiva estatisticamente significativa, ainda que fraca, entre coesão familiar e avaliação do funcionamento familiar resultante da entrevista clínica ( $R=0,40 ; P=0,027)$. A comparação com a adaptabilidade não mostrou resultados significativos ( $\mathrm{R}=0,24 ; P=0,195)$.

$\mathrm{O}$ estudo da escala BT demonstrou uma correlação linear forte e positiva com a avaliação clínica $(\mathrm{R}=0,91 ; P=$ 0,000). Observou-se, ainda, um resultado semelhante para a escala GARF $(\mathrm{R}=0,89 ; P=0,000)$ (tabela 4$)$.

\section{DISCUSSÃO}

A expectativa de que a escala FACES III provaria ser um instrumento diagnóstico de risco psiquiátrico útil para triagem em cuidados primários não se comprovou na prática quando se realizou a comparação com o padrão-ouro da avaliação clínica. Mesmo assim, ao compararmos nossos dados, obtidos com a aplicação da FACES III, com os resultados dos estudos internacionais de Olson e Córdoba, não observamos diferenças estatisticamente significativas quanto à proporção de indivíduos classificados nas diversas categorias de risco. A escala FACES III foi consagrada na literatura especializada por apresentar um comportamento coerente que se repetia em múltiplos estudos internacionais. A ausência de correlação entre a avaliação clínica e os resultados da escala FACES III, analisados pelo modelo circumplexo no estudo $\mathrm{A}$, demonstram a importância da testagem de instrumentos diagnósticos em relação a um padrão-ouro que comprove sua validade.

Além disso, o achado de uma correlação linear positiva entre a coesão familiar avaliada pela escala FACES III e a avaliada pelo padrão-ouro não foi suficientemente forte para apoiar seu uso como instrumento de triagem em cuidados primários. Cabe salientar que essa observação se refere a uma pequena amostra (32 famílias), sendo que uma análise da coesão e adaptabilidade utilizando o modelo linear poderia apresentar resultados diversos em uma amostra populacional maior.

Já os resultados observados na aplicação das escalas BT e GARF são muito promissores. As escalas demonstraram ser de simples manejo e aplicabilidade, com perspectivas de fácil treinamento para profissionais de saúde em cuidados primários, onde a avaliação do funcionamento familiar é tão necessária.

As três escalas, desenhadas para uso nos Estados Unidos, demonstraram ser interculturais ao adequarem-

TABELA 4. Comparação entre os resultados da aplicação das escalas diagnósticas e da entrevista clínica, Porto Alegre (RS), Brasil, 1997

\begin{tabular}{lcc}
\hline \multicolumn{1}{c}{ Escala } & $\begin{array}{c}\text { Coeficiente de } \\
\text { correlação (R) }\end{array}$ & $P^{\mathrm{a}}$ \\
\hline $\begin{array}{l}\text { Escala de coesão } \\
\text { do FACES IIIb }\end{array}$ & 0,40 & 0,027 \\
$\begin{array}{l}\text { Escala de } \\
\text { adaptabilidade do }\end{array}$ & & \\
$\begin{array}{l}\text { FACES III } \\
\text { Escala de Beavers- }\end{array}$ & 0,24 & 0,195 \\
$\begin{array}{l}\text { Timberlawn } \\
\text { Escala GARF }\end{array}$ & 0,91 & 0,000 \\
a Correlação linear. & 0,89 & 0,000 \\
\hline
\end{tabular}

${ }^{b}$ Análise através do modelo linear. 
se às características de uma população brasileira.

As escalas BT e GARF podem ser utilizadas em conjunto ou isoladamente. A escala BT, na nossa experiência, pareceu mais interessante para treinamento de pessoal a respeito da dinâmica e diagnóstico familiar, facilitando o registro do diagnóstico. A escala GARF é mais simples e seu uso parece muito adequado para o registro dos casos em cuidados primários. Ambas também podem ser úteis para a avaliação dos resultados do tratamento, quando são preenchidas no início e no final da intervenção.

\section{CONCLUSÕES}

Os resultados do presente estudo sugerem que as variáveis demográficas consideradas (número de filhos, idade do pai e da mãe, idade dos filhos e renda familiar) não interferem no funcionamento familiar. Além disso, nossos achados sugerem que a escala FACES III não é um instrumento adequado para a triagem de famílias de alto risco para doença mental em serviços primários de saúde. As escalas Beavers-Timberlawn e GARF, por sua vez, demonstraram ser adequadas para a formulação e registro do diagnóstico familiar e apropriadas para uso em atenção primária.

É evidente a importância do envolvimento da família na prática dos cuidados de saúde, tanto para a prevenção primária quanto para o diagnóstico e tratamento eficazes. É importante continuar a investir no estudo de instrumentos diagnósticos simples e práticos, de fácil treinamento, de valor intercultural, e que podem ser utilizados em todos os níveis de cuidados. A utilização de tais instrumentos pode, também, facilitar a realização de estudos comparativos internacionais que permitirão apreciar as semelhanças e as variações do funcionamento familiar nas diversas culturas.

A experiência dos autores aponta para a necessidade de desenvolver escalas auto-respondidas, ou de simples aplicação, de avaliação do funcionamento familiar, comparáveis à entrevista clínica, para identificar as muitas famílias que poderiam se beneficiar de uma atenção especial se suas dificuldades fossem identificadas nos serviços de cuidados primários.

A utilização de um instrumento padronizado para registrar os achados e a evolução dos resultados do tratamento tende a diminuir a subjetividade do avaliador, que é necessariamente parte do processo de avaliação. As escalas GARF e BT demonstraram ser muito adequadas para essa finalidade.

\section{REFERÊNCIAS}

1. Brink W, Leenstra A, Ormel J, Willige G. Mental health intervention programs in primary care: their scientific basis. J Affect Disord 1991;21(4):273-284.

2. Bowen M. Family therapy in clinical practice. New York: James Aronson Inc: 1978.

3. Minuchin S. Famílias, funcionamento e tratamento. Porto Alegre: Editora Artes Médicas; 1982.

4. Beavers WR. Healthy, midrange and severely dysfunctional families. Em: Walsh F. Normal family processes. New York: The Guilford Press; 1982. p. 45-66.

5. Falceto O, Aerts DRGC, Fernandes CL, Wartchow SE. O médico, o paciente e sua família. Em: Duncan B, Schmidt MI, Giugliani E. Medicina ambulatorial: condutas clínicas em atenção primária. Porto Alegre: Editora Artes Médicas; 1996. p. 65-76.

6. Pinsof WM, Wynne LC. The effectiveness and efficacy of marital and family therapy: intro- duction to the special issue. J Marital Family Therapy 1995;21:341-343. (Special issue on the effectiveness and efficacy of marital and family therapy).

7. American Psychiatric Association. Diagnostic and statistical manual of mental disorders (DMD-IV). 4a edição. American Psychiatric Association: Washington, DC; 1994.

8. Organização Mundial da Saúde. Classificação dos transtornos mentais e de comportamento da CID-10. Porto Alegre: Editora Artes Médicas; 1993.

9. Falceto O. Famílias com desenvolvimento funcional e disfuncional: validação das escalas diagnósticas FACES III, Beavers-Timberlawn e GARF [dissertação de mestrado]. Porto Alegre: Universidade Federal do Rio Grande do Sul: 1997.

10. Kaslow F. History, rationale and philosophic overview of issues and assumptions. Em: Kaslow F. Handbook of relational diagnosis and dysfunctional family patterns. New York: John Wiley \& Sons; 1996. p. 3-18.

11. Olson DH, Sprenkle DH, Russel CS. Circumplex model of marital and family systems: ICohesion and adaptability dimensons, family types and clinical applications. Fam Process 1979;18(1):3-28.

12. Olson DH, Sprenkle DH, Russel CS. Circumplex model: systemic assessment and treatment of families. New York: The Harworth Press; 1989.

Manuscrito recebido em 5 de abril de 1999. Aceito em versão revisada em 14 de dezembro de 1999. 
ANEXO 1. Instrumentos de coleta de dados, pesquisa sobre escalas diagnósticas do funcionamento familiar para utilização em serviços de atenção primária à saúde, Porto Alegre (RS) Brasil, 1997

\section{FACES III}

$\begin{array}{ccccc}1 & 2 & 3 & 4 & 5 \\ \text { Quase nunca } & \text { Raramente } & \text { Às vezes } & \text { Freqüentemente } & \text { Quase sempre }\end{array}$

DESCREVA SUA FAMÍLIA ATUALMENTE :

1. Os membros da família pedem ajuda uns aos outros.

2. Seguem-se as sugestões dos filhos na solução de problemas.

3. Aprovamos os amigos que cada um tem.

- 4. Os filhos expressam sua opinião quanto a sua disciplina.

5. Gostamos de fazer coisas apenas com nossa família.

— 6. Diferentes pessoas da família atuam nela como líderes.

- 7. Os membros da família sentem-se mais próximos entre si que com pessoas estranhas à família.

- 8. Em nossa família mudamos a forma de executar as tarefas domésticas.

- 9. Os membros da família gostam de passar o tempo livre juntos.

10. Pai(s) e filhos discutem juntos os castigos.

- 11. Os membros da família se sentem muito próximos uns dos outros.

12. Os filhos tomam as decisões em nossa família.

13. Estamos todos presentes quando compartilhamos atividades em nossa família.

- 14. As regras mudam em nossa família.

- 15. Facilmente nos ocorrem coisas que podemos fazer juntos, em família

- 16. Em nossa família fazemos rodízio das responsabilidades domésticas.

- 17. Os membros da família consultam outras pessoas da família para tomarem suas decisões.

- 18. É difícil identificar o(s) líder(es) em nossa família.

19. A união familiar é muito importante.

— 20. É difícil dizer quem faz cada tarefa doméstica em nossa casa.

POR FAVOR, INDIQUE SEU LUGAR NA FAMÍLIA :

MÃE__ PAI__ FILHO___ (LEMBRE QUE O FILHO MAIS VELHO CORRESPONDE AO № 1)

\section{ESCALA DE AVALIAÇÃO FAMILIAR DE BEAVERS-TIMBERLAWN}

I. ESTRUTURA DA FAMÍLIA

1. Evidências de distribuição de poder

\begin{tabular}{|c|c|c|c|c|}
\hline $\begin{array}{l}1 \\
\text { Caos }\end{array}$ & $\begin{array}{l}2 \\
\text { Marcada Dominação }\end{array}$ & $\begin{array}{l}3 \\
\text { Moderada Dominação }\end{array}$ & $\begin{array}{l}4 \\
\text { Liderada }\end{array}$ & $\begin{array}{l}5 \\
\text { Igualitária }\end{array}$ \\
\hline $\begin{array}{l}\text { Sem líder; ninguém tem } \\
\text { poder suficiente para } \\
\text { estruturar a interação. }\end{array}$ & $\begin{array}{l}\text { Controle próximo ao absoluto. } \\
\text { Não há negociação. } \\
\text { Dominação e submissão } \\
\text { são a regra. }\end{array}$ & $\begin{array}{l}\text { Controle próximo ao } \\
\text { absoluto. Alguma negociação, } \\
\text { mas dominação e submissão } \\
\text { são a regra. }\end{array}$ & $\begin{array}{l}\text { Tendência a haver } \\
\text { dominação e submissão, } \\
\text { mas a maioria das interações } \\
\text { é através de negociação } \\
\text { respeitosa. }\end{array}$ & $\begin{array}{l}\text { Liderança dividida entre os } \\
\text { pais, mudando com a natureza } \\
\text { da interação. }\end{array}$ \\
\hline \multicolumn{5}{|l|}{ 2. Coalizões parentais } \\
\hline $\begin{array}{l}11,5 \\
\text { Coalizão principal é entre } \\
\text { pai (ou mãe) e filho }\end{array}$ & 2,5 & $\begin{array}{l}3 \\
\text { Coalizão parental fraca }\end{array}$ & 4,5 & $\begin{array}{l}5 \\
\text { Coalizão parental forte }\end{array}$ \\
\hline \multicolumn{5}{|l|}{ 3. Intimidade } \\
\hline $\begin{array}{l}1,1,5 \\
\text { Limites entre os indivíduos } \\
\text { são amorfos e indistintos }\end{array}$ & 2,5 & $\begin{array}{l}3 \\
3\end{array}$ & 4,5 & $\begin{array}{l}5 \\
\text { Intimidade com limites } \\
\text { individuais bem distintos }\end{array}$ \\
\hline \multicolumn{5}{|c|}{ 4. Mitologia - Congruência da mitologia familiar (como a família define seu próprio funcionamento) com a realidade observável } \\
\hline $\begin{array}{l}1 \\
\text { Muito congruente }\end{array}$ & $\begin{array}{ll}2 & 2,5 \\
\text { Congruente na sua maior parte }\end{array}$ & 3,5 & $\begin{array}{l}4 \\
\text { Algo incongruente } \\
\end{array}$ & $\begin{array}{l}5 \\
\text { Muito incongruente }\end{array}$ \\
\hline \multicolumn{5}{|c|}{ 5. Capacidade de negociar e resolver problemas } \\
\hline $\begin{array}{lc}1 & 1,5 \\
\text { Extremamente eficiente }\end{array}$ & $\begin{array}{ll}2 & 2,5 \\
\text { Boa } & \end{array}$ & 3,5 & $\begin{array}{l}4 \\
\text { Pouco eficiente }\end{array}$ & $\begin{array}{l}5 \\
\text { Extremamente ineficiente }\end{array}$ \\
\hline \multicolumn{5}{|c|}{$\begin{array}{l}\text { II. AUTONOMIA } \\
\text { 6. Clareza da comunicação individual de pensamentos e sentimentos. }\end{array}$} \\
\hline Muito clara & 2,5 & $\begin{array}{l}3,5 \\
\text { Algo vaga e escondida }\end{array}$ & 4,5 & $\begin{array}{l}5 \\
\text { Quase nunca as pessoas } \\
\text { são claras }\end{array}$ \\
\hline
\end{tabular}


ANEXO 1. (Continuação)

\begin{tabular}{|c|c|c|c|c|c|c|c|}
\hline \multicolumn{8}{|c|}{ 7. Grau de responsabilidade assumida pelos membros sobre suas ações passadas, presentes e futuras. } \\
\hline $\begin{array}{l}1 \\
1,5 \\
\text { Em geral os membros } \\
\text { assumem responsabilidade } \\
\text { por suas ações individuais }\end{array}$ & 2 & 2,5 & & $\begin{array}{l}3,5 \\
\text { abros } \\
\text { dade } \\
\text { ais, m } \\
\text { acusa } \\
\text { a terce }\end{array}$ & 4 & 4,5 & $\begin{array}{l}5 \\
\text { Membros raramente ou nunca } \\
\text { assumem responsabilidade } \\
\text { por ações individuais }\end{array}$ \\
\hline \multicolumn{8}{|c|}{ 8. Invasão da individualidade do outro (falam um pelo outro, "lêem a mente" do outro) } \\
\hline $\begin{array}{l}1,5 \\
\text { Muitas invasões }\end{array}$ & 2 & 2,5 & $\begin{array}{l}3 \\
\ln \end{array}$ & $\begin{array}{l}3,5 \\
\text { ionais }\end{array}$ & 4 & 4,5 & $\begin{array}{l}5 \\
\text { Não há evidência de invasões }\end{array}$ \\
\hline \multicolumn{8}{|c|}{ 9. Receptividade, abertura e permeabilidade dos membros às comunicações dos outros. } \\
\hline $\begin{array}{ll}1 & 1,5 \\
\text { Muito grande }\end{array}$ & $\begin{array}{l}2 \\
M\end{array}$ & $\begin{array}{r}2,5 \\
\text { ite } \mathrm{gr}\end{array}$ & 3 & 3,5 & $\begin{array}{l}4 \\
\mathrm{~N} \\
\mathrm{n}\end{array}$ & $\begin{array}{l}4,5 \\
\text { ientemente } \\
\text { tivos }\end{array}$ & $\begin{array}{l}5 \\
\text { Membros não são receptivos }\end{array}$ \\
\hline
\end{tabular}

\section{AFETO DA FAMÍLIA}

10. Grau de expressividade dos vários tipos de sentimentos

\begin{tabular}{|c|c|c|c|c|}
\hline $\begin{array}{l}1 \\
\text { Expressão direta de grande } \\
\text { variedade de sentimentos }\end{array}$ & $\begin{array}{l}2 \\
\text { Expressão direta de muitos } \\
\text { sentimentos apesar de alguma } \\
\text { dificuldade }\end{array}$ & $\begin{array}{l}3 \\
\text { Restrição óbvia na expressão } \\
\text { de alguns sentimentos }\end{array}$ & $\begin{array}{l}4 \\
\text { Apesar de haver alguma } \\
\text { expressão de sentimentos, a } \\
\text { maioria é mascarada }\end{array}$ & $\begin{array}{l}5 \\
\text { A expressão de sentimento é } \\
\text { pequena ou inexistente }\end{array}$ \\
\hline \multicolumn{5}{|l|}{ 11. Tipo de afeto predominante } \\
\hline $\begin{array}{l}1 \\
\text { Em geral afetuosos, } \\
\text { com humor e otimismo }\end{array}$ & $\begin{array}{l}2 \\
\text { Polidos, com pouca } \\
\text { expressão de afeto ou calor, } \\
\text { freqüentemente hostis, } \\
\text { com algumas circunstâncias } \\
\text { de prazer }\end{array}$ & $\begin{array}{lr}3 & 3,5 \\
\text { Abertamente hostis }\end{array}$ & $\begin{array}{lc}4 & 4,5 \\
\text { Deprimidos (desvitalizados) }\end{array}$ & $\begin{array}{l}5 \\
\text { Cínicos, desesperançados e } \\
\text { pessimistas }\end{array}$ \\
\hline \multicolumn{5}{|c|}{ 12. Grau de conflito que parece impossível de resolver-se } \\
\hline $\begin{array}{l}1 \text { 1,5 } \\
\text { Conflito severo, com graves } \\
\text { problemas de funcionamento } \\
\text { do grupo }\end{array}$ & $\begin{array}{l}2 \\
2,5 \\
\text { Presença evidente de conflito, } \\
\text { com problemas de } \\
\text { funcionamento do grupo }\end{array}$ & $\begin{array}{l}3 \\
3,5 \\
\text { Presença evidente de conflito, } \\
\text { com leves problemas de } \\
\text { funcionamento do grupo }\end{array}$ & $\begin{array}{l}4 \quad 4,5 \\
\text { Algumas evidências de } \\
\text { conflito, sem problemas de } \\
\text { funcionamento do grupo }\end{array}$ & $\begin{array}{l}5 \\
\text { Pouco ou nenhum conflito } \\
\text { impossível de resolver-se }\end{array}$ \\
\hline \multicolumn{5}{|c|}{ 13. Empatia: grau de sensibilidade e compreensão dos membros em relação aos sentimentos dos outros } \\
\hline $\begin{array}{l}1,1,5 \\
\text { Respostas empáticas são } \\
\text { consistentes }\end{array}$ & $\begin{array}{l}2 \\
2 \\
\text { Maioria das respostas são } \\
\text { empáticas, apesar de haver } \\
\text { óbvia resistência }\end{array}$ & $\begin{array}{l}3 \text { 3,5 } \\
\text { Tentativa de envolvimento } \\
\text { empático, mas sem } \\
\text { consistência }\end{array}$ & $\begin{array}{l}4 \quad 4,5 \\
\text { Ausência de respostas } \\
\text { empáticas }\end{array}$ & $\begin{array}{l}5 \\
\text { Respostas obviamente } \\
\text { inapropriadas aos sentimentos } \\
\text { dos membros }\end{array}$ \\
\hline
\end{tabular}

\begin{tabular}{|c|c|c|c|c|c|c|c|c|}
\hline \multicolumn{9}{|c|}{ 14. ESCALA GLOBAL: Saúde-Patologia } \\
\hline $\begin{array}{c}1 \\
\text { Mais } \\
\text { sadias }\end{array}$ & 2 & 3 & 4 & 5 & 6 & 7 & 8 & $\begin{array}{cc}9 & 10 \\
& \text { Mais } \\
\text { patológicas }\end{array}$ \\
\hline
\end{tabular}

\section{ESCALA GARF}

(Avaliação Global do Funcionamento Interacional)

NOTA: Leia toda a escala cuidadosamente antes de dar sua avaliação. Use escores específicos, intermediários quando possível, p.ex. 45,68, 72. Se não há informação detalhada adequada para dar escores específicos, use pontuações médias nas cinco partes, isto é, $90,70,50$ ou 10 .

5. (81-99) Existem padrões e rotinas combinados que permitem a satisfação das necessidades habituais de cada participante; existe flexibilidade para mudar a resposta a eventos ou necessidades fora do usual; conflitos ocasionais e transições difíceis são resolvidos através de comunicações e negociações destinadas a solucionar problemas.

Existe um entendimento compartido e acordo sobre os papéis e tarefas apropriados; a tomada de decisões é estabelecida para cada área funcional; existe reconhecimento das características particulares e dos méritos de cada subsistema (p.ex. pais/casal, irmão e indivíduos).

Existe uma atmosfera otimista nas relações apropriada para a situação; uma grande variedade de sentimentos é livremente expressa e elaborada; há uma atmosfera geral de calor, carinho e valores compartidos. As relações sexuais dos adultos são satisfatórias.

EM SUMA: A unidade interacional está funcionando satisfatoriamente segundo o relato dos participantes e a perspectiva dos observadores. 


\section{ANEXO 1. (Continuação)}

4. (61-80) A maioria dos problemas interacionais corriqueiros é resolvida adequadamente, mas existe dor e dificuldade em responder a situações incomuns. Alguns conflitos permanecem não resolvidos, mas não perturbam a relação.

A tomada de decisões é feita, em geral, de forma competente, mas o esforço para o controle dos membros entre si, às vezes, é maior que o necessário e/ou é inefetivo. Indivíduos e coalizões são claramente demarcados mas, às vezes, são depreciados ou discriminados.

Uma gama de sentimentos é expressa, mas é evidente que há áreas de bloqueio emocional e tensão. Calor e carinho estão presentes, mas são marcados por irritabilidade e frustração. A atividade sexual dos adultos pode ser algo insatisfatória e problemática.

EM SUMA: O funcionamento da unidade interacional é algo insatisfatório. São resolvidas muitas das dificuldades que ocorrem ao longo do tempo, mas não todas elas.

3. (41-60) A comunicação, a solução de problemas e as atividades rotineiras, com bastante freqüência, são inibidas ou atrapalhadas por conflitos não resolvidos; há dificuldade moderadamente grave em adaptar-se a situações de stress e transições, como saídas da família, mortes, nascimentos e casamentos.

A tomada de decisões é só intermitentemente competente e efetiva; nessas situações observa-se excessiva rigidez ou falta significativa de estrutura. As necessidades individuais estão freqüentemente submersas.

Dor e/ou raiva inefetiva ou paralisia emocional interferem com a possibilidade de compartir alegrias. Apesar de haver algum calor e apoio para os membros, esses, em geral, são desigualmente distribuídos. Problemas sexuais entre os adultos são freqüentes.

EM SUMA: Apesar de haver períodos ocasionais de funcionamento satisfatório e competente das relaçóes, aquelas disfuncionais e insatisfatórias tendem a prevalecer.

2. (21-40) Os padrões e rotinas interacionais não satisfazem as necessidades dos membros; expectativas estabelecidas são ignoradas ou rigidamente cumpridas, apesar de mudanças situacionais. Transições do ciclo vital como partidas ou entradas das/nas relações geram problemas frustrantes e não resolvidos.

A tomada de decisões é tirânica ou bastante ineficaz. As características particulares dos indivíduos não são apreciadas, ou são ignoradas por coalizões rígidas ou confusamente fluidas.

Períodos de convivência agradável em conjunto são infreqüentes; distância óbvia e hostilidade declarada refletem conflitos importantes que permanecem não resolvidos e bastante doídos. Disfunção sexual grave entre os adultos é freqüente.

EM SUMA: A unidade interacional é óbvia e seriamente disfuncional. Períodos de relacionamento satisfatório são raros.

1. (1-20) As rotinas interacionais são poucas (p.ex., não há horários combinados de refeições, sono ou período de vigília); os membros da casa freqüentemente não sabem onde os outros estão, ou o que esperar uns dos outros; a comunicação é repetidamente atrapalhada por mal-entendidos e falta de atenção no que os outros dizem.

Responsabilidades pessoais e geracionais não são reciprocamente aceitas e reconhecidas. Os limites da unidade interacional como um todo e dos subsistemas não podem ser identificados ou respeitados. Pessoas, nessa relação, podem fisicamente ameaçar, agredir ou sexualmente atacar umas às outras.

O desespero e o cinismo são francos; pouca atenção é prestada às necessidades emocionais dos outros; quase não existe sentimento de pertencimento, ligação ou preocupação com o bem-estar uns dos outros.

SUMA: A unidade interacional tornou-se excessivamente disfuncional para garantir a continuidade de contato e ligação.

0. Informação inadequada.

ESCORE ATUAL

MAIS COMPETENTE NO ANO PASSADO MENOS COMPETENTE NO ANO PASSADO NO INÍCIO DO TRATAMENTO

Adequação da escala (marque uma das opções)

1. não aplicável

2. difícil (caso compatível com dois ou mais níveis)

3. pobre (possível, mas as características principais do caso não combinavam)

4. bastante boa

5. muito boa

6. informação inadequada para classificar

Comente livremente a impressão que essa família Ihe deixou: 
ABSTRACT Care provided to patients in primary health services should include both a physical and a psychological assessment. In many cases treatment success depends on understanding individuals and their interactions, especially within the family; it is important for health professionals to be trained in this area. We investigated the validity of three well-known diagnostic scales of family functioning from the United States of America (FACES III, Beavers-Timberlawn, and GARF) for use in our setting in Porto Alegre, Brazil. The three scales were used with 31 families and compared to the results from clinical evaluations (semistructured family interviews), which were considered the gold standard. FACES III was also used with a sample of 102 families in the community. The Beavers-Timberlawn and GARF scales were strongly and positively correlated with the clinical evaluations. In contrast, our results suggest that FACES III is not a useful screening tool in primary care to detect the risk of psychiatric disorders. The Beavers-Timberlawn and GARF instruments showed great usefulness for formulating and classifying diagnoses of family functioning.

\begin{tabular}{|c|}
\hline I Simposio Internacional sobre Género y Salud \\
\hline $\begin{array}{l}\text { Fechas: } 25 \text { al } 28 \text { de octubre de } 2000 \\
\text { Lugar: } \quad \text { Santiago de Cuba } \\
\text { Tema: } \quad \text { La perspectiva de género: una necesidad para el desarrollo humano } \\
\text { La Sociedad Cubana de Medicina Familiar convoca a todos los profesionales, institucio- } \\
\text { nes y organizaciones de salud a participar en este simposio auspiciado por el Ministerio de } \\
\text { Salud Pública de Cuba, la OMS/OPS y otras entidades internacionales y nacionales. El simpo- } \\
\text { sio tiene por objetivos explorar mecanismos para incorporar la perspectiva de género en las ini- } \\
\text { ciativas de desarrollo local y en la atención integral a la salud reproductiva, así como discutir } \\
\text { la adopción de políticas de salud para el bienestar de la mujer, el niño y el adolescente y con- } \\
\text { tribuir a la perspectiva de género en la formulación de estas políticas. Se hará hincapié en las } \\
\text { experiencias de Cuba y otros países y de los organismos internacionales en materia de desarro- } \\
\text { Ilo municipal desde una perspectiva de género y se examinarán las principales dificultades que } \\
\text { enfrentan los sistemas de salud en este campo. Habrá visitas a diversos consultorios y policlí- } \\
\text { nicos y al Sistema Nacional de Salud, donde se podrá ver en la práctica cómo funciona el sis- } \\
\text { tema sanitario en Cuba. } \\
\text { Información: } \\
\text { Dra. Clarivel Presno Labrador } \\
\text { Presidenta, Sociedad Cubana de Medicina Familiar } \\
\text { Fax: (537) } 335920 \\
\text { (537) } 662075\end{array}$ \\
\hline
\end{tabular}

